\title{
Experimental study of the dynamic behaviour of High Performance Concrete (HPC) under tensile loading
}

\author{
Bratislav Lukic ${ }^{1, *}$, Dominique Saletti ${ }^{1}$, and Pascal Forquin ${ }^{1}$ \\ ${ }^{1}$ Univ. Grenoble Alpes, 3SR, F-38000 Grenoble, France
}

\begin{abstract}
This paper presents the measurement results of the dynamic tensile strength of a High Performance Concrete (HPC) obtained using full-field identification method. An ultra-high speed imaging system and the virtual fields method were used to obtain this information. Furthermore the measurement results were compared with the local point-wise measurement to validate the data pressing. The obtained spall strength was found to be consistently $20 \%$ lower than the one obtained when the Novikov formula is used.
\end{abstract}

\section{Introduction}

Owing to its elevated mechanical properties, High Performance Concrete (HPC) presents an affordable alternative to ordinary concrete material. This is especially the case when engineering structures are designed to resist severe dynamic loading such as blasts or impact under which complex failure phenomena take place [1]. In order to improve its applications in engineering design of protective structures, the dynamic properties of HPC need to be well examined and supported by throughout collection of reliable experimental data on its failure characteristics. This is particularly necessary in dynamic tension, since concrete materials exhibit sensitivity to high strain rates, namely the increase of tensile strength with the increase of the loading rate [2].

Presently, the dynamic tensile behaviour of HPC is investigated via the spalling technique [3]. An innovative full-field method based on the use of ultra-high-speed photography and the virtual fields method (VFM) is applied to process the data [4]. The VFM provides the direct relation between measured acceleration field and the average axial stress in the cross-section by exploiting the samples intentional non-equilibrium state throughout the test. This then allows obtaining local stress-strain response at any cross- section along the sample axis which is visualised with the ultra-high-speed (UHS) camera. The identification technique has been validated using simulated experiments by the means of synthetic image processing in some previous works [5] and has been applied to processing several spalling experiments on ordinary concrete [6-7]. The tested HPC samples in this work are instrumented with a grid pattern and the grid method was used to obtain time- resolved full-field displacement maps on the sample surface [11]. Alongside full field measurements, conventional pointwise measurements (strain gauges and laser extensometer) were used. The local information obtained from employing virtual gauge processing of full field data is compared to local measurements. Finally, the failure tensile stress obtained from several spalling tests using both the identification from full-field measurements and the local measurement of the rearface velocity pull-back based on the Novikov processing [8], are reported and compared.

\section{Experimental methodology}

\subsection{The photomechanical spalling set-up}

The tests were performed using the spalling test setup in Laboratory 3SR (Figure 1). It consists of a single in- put Hopkinson bar which is in contact with a cylindrical sample on one end which is impacted by a cylindrical projectile on the other end. In that way, a compressive pulse is generated which travel towards the sample and reflects from the sample's free-end into a tensile wave which causes the failure of the concrete sample in tension. The material used for the bar and the projectile is an aluminium alloy of the following characteristics: density $2810 \mathrm{~kg} / \mathrm{m}^{3}$, expected elastic Young's modulus $72 \mathrm{GPa}$, material wave speed $\mathrm{C}_{0}=5090 \mathrm{~m} / \mathrm{s}$ and high yielding strength of $450 \mathrm{MPa}$. The input bar is $1.4 \mathrm{~m}$ in length and $45 \mathrm{~mm}$ in diameter. It is instrumented with two crossed-element standard foil strain gauges connected into a full bridge circuit that is located in its mid-length. The concrete samples were instrumented with three standard foil strain gauges and have been positioned at $40 \mathrm{~mm}, 60 \mathrm{~mm}$ and $120 \mathrm{~mm}$ from the freeend. All gauges were connected to a high resolution data acquisition control apparatus with a $1 \mathrm{MHz}$ registration frequency. A laser interferometer, having a bandwidth of 1.5 $\mathrm{MHz}$, was pointed towards the sample free-end to register the particle velocity. The grid instrumented 
sample surface was recorded with the Shimadzu HPV$\mathrm{X} 2$ camera. The imaging system provides 128 consequently capture frames of $400 \times 250$ pixel count with 10-bit dynamic range. Thanks to increased sensitivity of the HPV-X2 [9], the sample surface was illuminated using two DedoCool tungsten light sources, operating on boost mode with $250 \mathrm{~W}$. The deposited grid pattern on samples' surfaces has $1 \mathrm{~mm}$ pitch and was filmed using acquisition speeds of both $1 \mathrm{Mfps}$ and 2 Mfps and 7 pixels/period grid sampling.

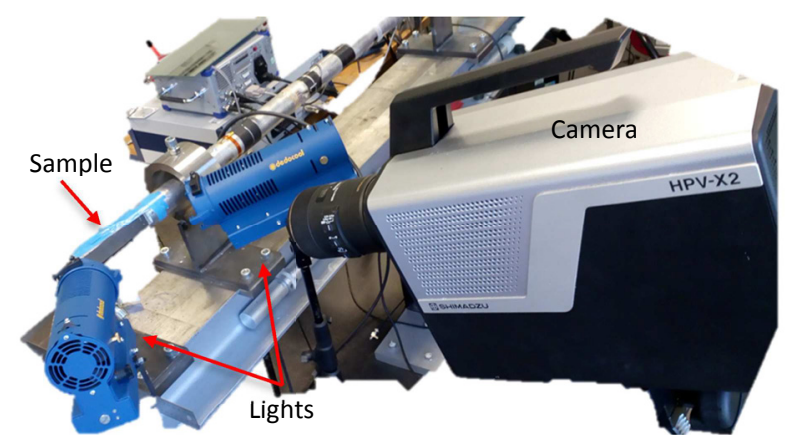

Fig. 1. The photomechanical set-up used to perform the spalling tests.

\subsection{Tested material}

High performance concrete (HPC) used in this work is characterized by strong cement matrix and lower capillary porosity when compared to ordinary concrete. The mixture was designed with the aim of investigating the effect of cement matrix porosity on concrete confined response [10]. Reduced porosity is achieved by adding silica fumes and decreasing the water-cement ratio. Having this ratio set to 0.3 , the expected uniaxial compressive strength after 28 days is $80 \mathrm{MPa}$. The quantity of sand was adjusted for workability reasons and the mixture is also characterized by the rolled siliceous aggregate with the maximum aggregate size of $8 \mathrm{~mm}$. The composition and the mix proportions are given in Table 1.

Table 1. Composition of the HPC mixture used in dynamic tensile tests [10].

\begin{tabular}{|l|r|}
\hline Concrete & HPC \\
\hline Aggregates D 0.5/8 $\left[\mathrm{kg} / \mathrm{m}^{3}\right]$ & 1008 \\
Sand $\mathrm{D}_{\max }=1800 \mu \mathrm{m}\left[\mathrm{kg} / \mathrm{m}^{3}\right]$ & 795.4 \\
Cement CEM I $52.5 \mathrm{~N}$ PM ES CP2 (Vicat) & 420 \\
{$\left[\mathrm{~kg} / \mathrm{m}^{3}\right]$ Superplasticizer (Sikafluid) $\left[\mathrm{kg} / \mathrm{m}^{3}\right]$} & 4.7 \\
Silica fumes (Condensil S95 DS) $\left[\mathrm{kg} / \mathrm{m}^{3}\right]$ & 46.7 \\
Water $\left[\mathrm{kg} / \mathrm{m}^{3}\right]$ & 140 \\
\hline Uniaxial compressive strength at 28 days $(\mathrm{MPa})$ & 80 \\
Water/cement ratio (-) & 0,3 \\
\hline
\end{tabular}

\subsection{The VFM applied to a spalling test (stress gauge)}

The VFM is a suitable identification strategy when the full field kinematic fields are measured in the region of interest [12]. The procedure for identifying the evolution of axial stress along the sample axis throughout the test is based on the analysis presented in [4]. From the weak form of the dynamic equilibrium equation, it is possible to reconstruct the average axial stress in any visualized cross-section along the sample axis $\mathrm{x}$ by averaging the longitudinal acceleration over the area between the considered cross section at position and the free end (Figure 2). The resulting description for the axial stress can be expressed as:

$$
\overline{\sigma(x, t)}=-\rho b(x) \overline{a_{x}(x, t)}
$$

where $\overline{\sigma(x, t)}$ is the average axial stress in the crosssection at the observed position $x, \rho$ is the material density, $b(x)$ is the longitudinal distance from the sample free end to the observed cross-section and $\overline{a_{x}(x, t)}$ is the average surface longitudinal acceleration component considering the area between the observed cross-section at position $x$ and the free end.

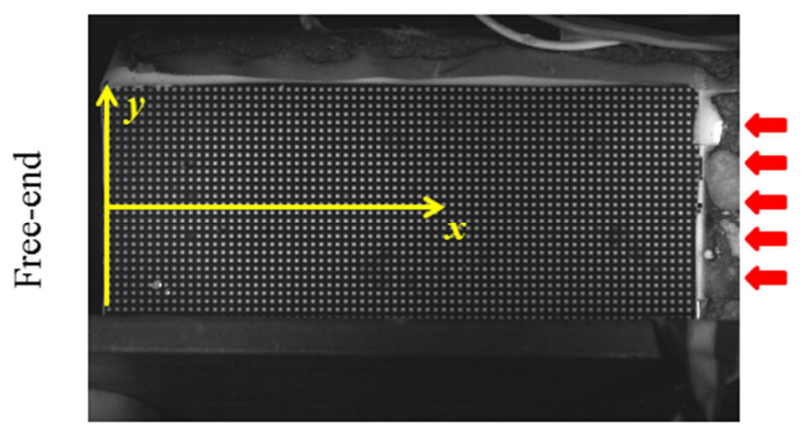

Fig. 2. Image of a specimen with $1 \mathrm{~mm}$ pitch grid pattern taken with a Shimadzu HPV-X2 camera at 2 Mfps.

\section{Experimental results}

\subsection{The identification results}

The obtained time-resolved displacement fields are processed to derive the sought identification results. The axial strain maps are derived from the displacement fields using the diffuse approximation approach [13] with 5 spatial point kernel while the velocity fields were obtained by a temporal derivation of displacement signals using a local $2^{\text {nd }}$ order polynomial fit over 11 time points. The acceleration fields are obtained from the velocity fields using one additional step of temporal derivation. In order to verify the processing parameters the local measurements are compared to those obtained using the full field data. The comparison of the axial strain measurement using the strain gauge and the virtual strain gauge from the full-field measurements is presented in Figure 3 and the comparison of the rear-face velocities is shown in Figure 4. A good agreement 
between the results obtained with these two types of measurement systems can be observed.

\subsection{Identified tensile strength and strain rate}

The peak tensile strength of six tested HPC samples is obtained from the reconstructed local stress-strain response at positions where discontinuities were observed in the measured displacement maps. The axial stress in the sample was obtained directly from the acceleration fields without assumption on the material constitutive behaviour using Equation 1. The fields of axial strain rate were obtained by performing a temporal derivation of the axial strain maps. The strain rate at failure was obtained from the virtual gauge processing and the measured time to peak tensile stress as reported in [6]. The peak tensile stress was also calculated from the measurement of rear face velocity profile as:

$$
\sigma_{t}=\frac{1}{2} \rho C_{0} v_{p b}
$$

where $\rho$ is the material density, $C_{0}$ is the material wave speed and the $v_{p b}$ is the pull-back velocity, corresponding to the difference between the peak velocity and the velocity at the first rebound.

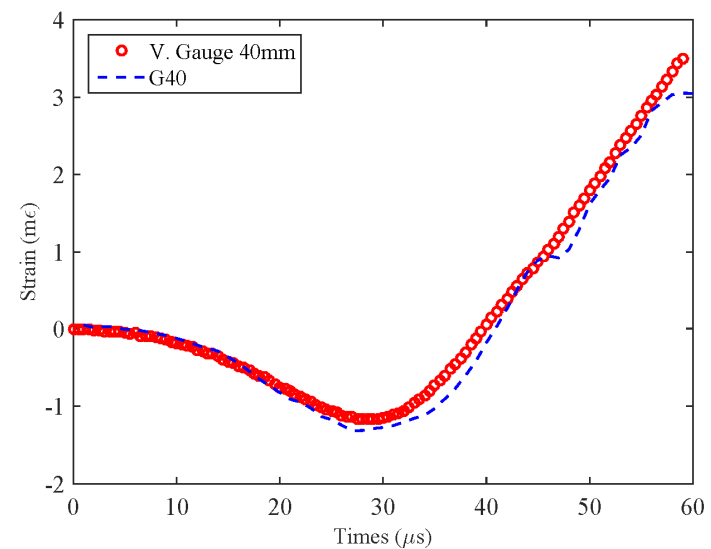

Fig. 3. Comparison of the axial strain obtain using strain gauge and virtual strain gauge derived from the full-field measurements at $40 \mathrm{~mm}$ from sample free-end.

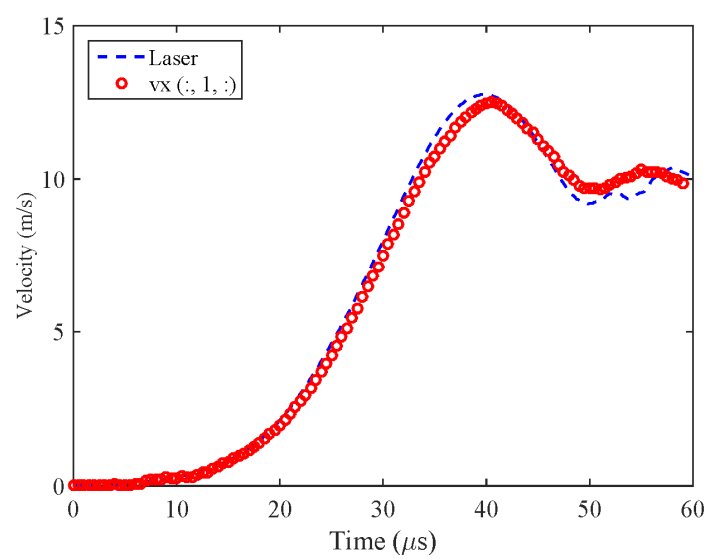

Fig. 4. Comparison of the rear-face velocity obtain using laser interferometer and the full-field measurements from Shimadzu HPV-X2 camera at 2 Mfps.

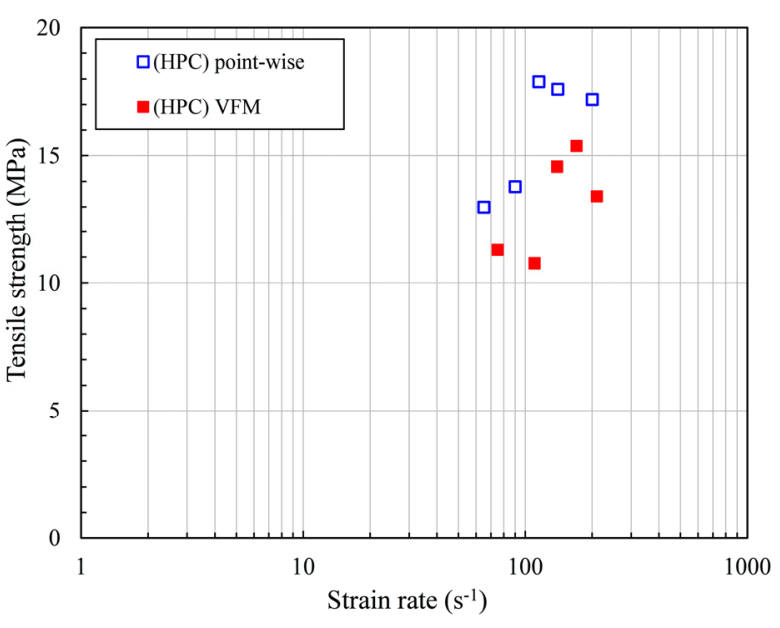

Fig. 5. Image of a specimen with $1 \mathrm{~mm}$ pitch grid pattern taken with a Shimadzu HPV-X2 camera at 2 Mfps.

The comparison between the identified peak tensile stress using the VFM identification and the stress at failure obtained using the velocity pull-back with respect to strain rate is shown in Figure 5. From the presented plot it can be observed that the classical method shows higher dynamic tensile strength of about $20 \%$ when compared to the values identified from full field measurements. The main reasons for this over-estimate, as discussed in [6], is certainly coming from strong assumptions used in the classical processing based on pull-back velocity. Namely, the assumptions of perfect elasticity up-to the peak and the unitary value of Young's modulus in tension and compression.

\section{Conclusions}

In this work the tensile strength of HPC is determined with two methods. The first one uses the VFM to process the full field displacement data supplied through an UHS imaging system and the second one relies on the classical Novikov processing of the pull-back velocity. The dynamic tensile strength was found to be consistently lower in case when the VFM is used. The presented results clearly indicate that the strong assumptions made during the traditional Novikov processing of the experiments using the rear-face velocity pull-back can lead to over-estimated results regarding the tensile strength of quasi-brittle materials.

The authors would like to express their gratitude to LMT Cachan for supplying the ultra-high-speed acquisition system.

\section{References}

1. Q.M. Li, S.R. Reid, H.M. Wen, A.R. Telford, Int. J. Imp. Eng. 32, 1-4 (2005)

2. L. J. Malvar, C. A. Ross, ACI. Mat. J. 95 (1998)

3. B. Erzar, P. Forquin, Exp. Mech. 50, 941-955 (2010)

4. F. Pieron, P. Forquin, Strain 48, 388-405 (2012)

5. B. Lukic, D. Saletti, P. Forquin, Philos. Trans. Royal Soc. A 375 (2017) 
6. P. Forquin, B. Lukic, J. Dyn. Behav. Mater. 4, 34-55 (2018)

7. B. Lukic, D. Saletti, P. Forquin, J. Dyn. Behav. Mater. 4, 55-73 (2018).

8. S. A. Novikov, I.I. Divinov A.G. Ivanov, Fiz. Met. i Metalloved 21, 122-128 (1966)

9. R. Kuroda, S. Sugawa, Proc. SPIE 10328, Sel. Pap. from $31^{\text {st }}$ Int. Congr. High-Speed Imaging Photonics, Osaka, Japan, 1-6 (2017)
10. L. Zingg, M. Briffaut, J. Baroth, Y. Malecot, Cem. Concr. Res. 80, 52-59 (2016)

11. M. Grédiac, F. Sur, B. Blaysat, Strain 52, 205-243 (2016)

12. F. Pieron,M. Grédiac, The Virtual Fields Method (Springer, NY, 2012)

13. S. Avril, P. Feissel, F. Pierron, P. Villon, Eur. J. Com- put. Mech. 17, 857-868 (2008) 